\title{
Caractérisation de la pollution bactériologique des effluents de la ville de Nouakchott, irrigués dans le périmètre maraîcher de Sebkha
}

\author{
Abdoulaye Demba N'DIAYE ${ }^{1 *}$, Mohamed Ould Sid'Ahmed Ould KANKOU ${ }^{2}$, \\ Baidy LO $^{1}$ et Khalid IBNO NAMR ${ }^{3}$ \\ ${ }^{1}$ Laboratoire de Chimie de l'Eau, Institut National de Recherches en Santé Publique de Nouakchott, BP 690, \\ Tél. (222) 52531 75, Mauritanie. \\ ${ }^{2}$ Laboratoire de Chimie de l'Eau et Environnement, Faculté des Sciences et Techniques de l'université de \\ Nouakchott- BP 5026, Tél. (222) 52513 82, Mauritanie. \\ ${ }^{3}$ Laboratoire des Sciences du Sol et Environnement, Département de Géologie, Faculté des Sciences BP. 20 - \\ El Jadida 24 000, Tél : 212 (0) 523342325 /343 003, Maroc. \\ *Auteur correspondant, E-mail: abdouldemba@yahoo.fr
}

\section{RESUME}

La présente étude se propose d'une part de suivre l'évolution des paramètres physicochimiques et bactériologiques des effluents de la ville de Nouakchott au cours des mois de février, mars et avril 2009, et d'autre part d'évaluer ces données avec des méthodes statistiques. Les paramètres physicochimiques étudiés sont la température, le $\mathrm{pH}$, la conductivité électrique, des chlorures, ammonium et orthophosphates. Les paramètres bactériologiques sont les coliformes fécaux et streptocoques fécaux. Les campagnes d'analyses réalisées ont démontré que les niveaux de pollutions bactériologiques sont supérieurs aux seuils recommandés par l'O.M.S. Les teneurs maximales en coliformes fécaux et streptocoques fécaux peuvent atteindre respectivement 9,3.10 $\mathrm{UFC} / 100 \mathrm{ml}$ et $9,6.10^{4} \mathrm{UFC} / 100 \mathrm{ml}$. Le rapport $\mathrm{CF} / \mathrm{SF}$ est compris entre 0,7 et 1 , ce qui laisse supposer que les effluents de la ville de Nouakchott sont contaminés par une pollution très probablement mixte (d'origine animale et humaine) avec une prédominance animale. L'application de l'analyse en composante principale nous renseigne que la pollution fécale est d'origine domestique.

(C) 2011 International Formulae Group. All rights reserved.

Mots clés : Fécal, Moughataa, Tevragh Zeina, Mauritanie.

\section{INTRODUCTION}

Au cours du IXX ${ }^{\mathrm{e}}$ siècle, les maladies d'origine hydrique ont été responsables de vastes épidémies de dysenterie, fièvre typhoïde, choléra, entre autres (Angelo et al., 1997 ; Balbus et al., 2002). Aujourd'hui, ces maladies sont à l'origine d'un taux de mortalité très élevé des populations des pays en voie de développement. Ces maladies sont le plus souvent transmises par voie féco-orale, et la contamination de l'homme se réalise soit par consommation d'eau de boisson, soit par consommation d'aliments contaminés par l'eau, soit encore lors d'un bain ou d'un contact avec des eaux à usage récréatif (George et Servais, 2002).

Le périmètre maraîcher de Sebkha a fait l'objet de nombreux travaux (Azandosessi et 
al., 1997 ; Ould Baba, 1998 ; N'diaye et al., 2009). Il a été souligné que le périmètre est soumis à une forte pollution par les effluents de la ville de Nouakchott. Ces effluents contiennent de nombreux organismes pathogènes, représentant une menace pour la santé humaine. L'estimation de la contamination se fait par le biais des bactéries indicatrices de pollution fécale et de germes pathogènes. Ces indicateurs bactériens sont les coliformes fécaux (CF) et les streptocoques fécaux (SF). Dans le but d'estimer le risque de contamination fécale et de caractériser la charge fécale contenue dans les effluents de la ville de Nouakchott, nous avons utilisé l'Analyse en Composante Principale (ACP) couplée avec quelques paramètres physicochimiques.

\section{MATERIEL ET METHODES}

\section{Milieu et site d'étude}

Nouakchott est une ville côtière (superficie de $400 \mathrm{~km}^{2}$ ) située vers $18^{\circ} 07$ de latitude nord et $16^{\circ} 01$ de longitude ouest, est géographiquement ouverte sur la façade atlantique. Le climat y est généralement sec toute l'année avec des pluies faibles et très irrégulières durant l'été. Les températures oscillent entre $28,4{ }^{\circ} \mathrm{C}$ et $36,4{ }^{\circ} \mathrm{C}$ pour les maxima et $14,6{ }^{\circ} \mathrm{C}$ et $25,7{ }^{\circ} \mathrm{C}$ pour les minima (Mint El Bezeid, 2006). On a choisi comme site du prélèvement la STEP (Station de Traitement des Eaux Polluées) afin d'obtenir une mesure représentative sur l'ensemble des eaux usées drainées par certains quartiers de la ville de Nouakchott et de quelques unités industrielles connectées aussi à la STEP (SOBOMA : Société de Boisson de Mauritanie et la Pro Pêche, une société de pêche). La SOBOMA est équipée de plusieurs machines telle que la chaudière, la soutireuse, la laveuse, etc. la soutireuse fonctionne en parallèle avec le rinçage des bouteilles qui passent pour le remplissage et le sertissage. Les températures des bains de rinçage des bouteilles se situent dans un intervalle de 20 ${ }^{\circ} \mathrm{C}$ à $80{ }^{\circ} \mathrm{C}$. Au niveau de la SOBOMA, la soude caustique est le principal constituant employé pour le nettoyage des bouteilles dont la concentration varie entre 0 et $3 \%$ selon les bains. La Pro Pêche est spécialisée dans le domaine des céphalopodes (poulpes, seiches). Pour le traitement des céphalopodes, la Pro Pêche utilise de l'eau de mer. Le réseau fonctionnel est d'une longueur de $38 \mathrm{~km}$ environ, desservant le centre ville et la Moughataa de Tevragh Zeina.

\section{Méthodes d'analyses}

Les prélèvements hebdomadaires ont lieu au cours des mois de février, mars et avril 2009. Les échantillons d'eau usée sont prélevés dans des flacons en polyéthylène de 1 litre. Les analyses ont lieu immédiatement après prélèvement, au Laboratoire de Chimie de l'Eau de l'INRSP (Institut National de Recherches en Santé Publique). Les paramètres physicochimiques étudiés sont: la température, le $\mathrm{pH}$, la conductivité, les ions chlorures, les ions ammonium et les ions orthophosphates. Les paramètres bactériologiques sont les coliformes fécaux et les streptocoques fécaux.

Le $\mathrm{pH}$ et la température ont été déterminés par un pH-mètre de type Hanna muni d'une sonde mesurant la température. La conductivité a été mesurée par un conductimètre de type Hanna. Les chlorures sont mesurés par méthode de Mohr. Les ions ammonium et orthophosphates sont analysés par des méthodes colorimétriques à l'aide d'un spectrophotomètre UV Visible de type 722 S Beijing, les ions ammonium par le réactif Nessler. Pour le dosage des orthophosphates, on utilise un réactif molybdique. Pour le dénombrement des coliformes fécaux et les streptocoques fécaux, nous avons utilisé la technique des dilutions-étalements. Le milieu de culture utilisé pour les coliformes fécaux est la gélose Mac Conkey, et le dénombrement des colonies est effectué après 24 heures d'incubation à $44{ }^{\circ} \mathrm{C}$. L'isolement des streptocoques fécaux est effectué par la même méthode, mais sur le milieu de culture (gélose Slanetz) incubé à $37{ }^{\circ} \mathrm{C}$ pendant 48 heures. 
Les résultats sont exprimés par Unité Formant Colonie par $100 \mathrm{ml}$ (UFC/100 ml). L'étude statistique a été basée sur l'ACP. Les matrices de corrélations intermédiaires et les corrélations entre les variables et les axes et les projections des variables dans l'espace des axes F1et F2 ont été obtenus avec un Logiciel XLSTAT 2010.

\section{RESULTATS}

Aspect qualitatif des effluents de la ville de Nouakchott

Le Tableau 1 donne les valeurs maximales, minimales, moyennes et les écarts types de quelques paramètres physicochimiques étudiés au cours des trois mois (février, mars et avril 2009). Le Tableau 2 donne les valeurs maximales, minimales, moyennes et les écarts types des Coliformes fécaux et Streptocoques fécaux durant les trois mois (février, mars et avril) 2009. Dans les effluents de la ville de Nouakchott, les teneurs moyennes en coliformes fécaux et streptocoques fécaux sont respectivement $8,6.10^{4} \mathrm{UFC} / 100 \mathrm{ml}$ et $8,9.10^{4} / 100 \mathrm{ml}$.

\section{Application de l'analyse en composante principale}

Le traitement des données par l'analyse en composante principale, en utilisant comme variables la température, le $\mathrm{pH}$, la conductivité, les ions chlorures, des ions ammonium, des ions orthophosphates, les coliformes fécaux et les streptocoques fécaux et comme individus, les 13 prélèvements effectués au niveau de la STEP. On donne dans le Tableau 3 les coefficients de corrélations entre les variables et les deux premiers axes. Ces axes montrent une bonne répartition et représentation des variables étudiés. Le Tableau 4 donne les corrélations entre les variables et les facteurs. La Figure 1 donne les projections des variables dans l'espace des axes F1et F2.

Tableau 1 : Résultats des paramètres physicochimiques étudiés.

\begin{tabular}{lllllll}
\hline Paramètres & $\mathbf{T}$ & $\mathbf{p H}$ & $\mathbf{C E}$ & $\mathbf{C l}^{-}$ & $\mathbf{N H}_{\mathbf{4}}{ }^{+}$ & $\mathbf{P O}_{\mathbf{4}}{ }^{3-}$ \\
\hline Unités & ${ }^{\circ} \mathrm{C}$ & & $\mathbf{m S} / \mathbf{c m}$ & $\mathbf{~ m g / l i t r e}$ & $\mathbf{m g} /$ litre & $\mathbf{m g / l i t r e}$ \\
Min. & 21,9 & 6,91 & 1,5 & 194 & 119,14 & 19,81 \\
Max. & 26,3 & 8,17 & 5,5 & 1633 & 292,05 & 39,15 \\
Moy. & 24,3 & 7,42 & 3,17 & 789,84 & 209,47 & 28,35 \\
Ecart-types & 1,58 & 0,37 & 1,26 & 433,09 & 47,81 & 5,09 \\
\hline
\end{tabular}

Tableau 2 : Résultats bactériologiques des effluents de la ville de Nouakchott.

\begin{tabular}{llllll}
\hline Variables & Unité & Min. & Max. & Moy. & Ecart-type \\
\hline CF & UFC/100 ml & $7,1.10^{4}$ & $9,3.10^{4}$ & $8,6.10^{4}$ & 1035 \\
SF & UFC/100 ml & $8.10^{4}$ & $9,6.10^{4}$ & $8,9.10^{4}$ & 655 \\
\hline
\end{tabular}

Tableau 3 : Corrélations entre les variables et les axes principaux.

\begin{tabular}{lcc}
\hline & F1 & F2 \\
\hline $\mathbf{T}$ & 0,737 & 0,660 \\
$\mathbf{p H}$ & 0,859 & 0,512 \\
$\mathbf{C E}$ & 0,918 & 0,388 \\
$\mathbf{C l}-$ & 0,818 & 0,573 \\
$\mathbf{N H}_{4}{ }^{+}$ & $-0,678$ & 0,695 \\
$\mathbf{P O}_{4}{ }^{3-}$ & $-0,637$ & 0,725 \\
$\mathbf{C F}$ & $-0,514$ & 0,787 \\
$\mathbf{S F}$ & $-0,488$ & 0,849 \\
\hline
\end{tabular}


Tableau 4 : Matrice des corrélations inter-élémentaires.

\begin{tabular}{lllllllll}
\hline Variables & $\mathbf{T}$ & $\mathbf{p H}$ & $\mathbf{C E}$ & $\mathbf{C l}-$ & $\mathbf{N H}_{\mathbf{4}}{ }^{+}$ & $\mathbf{P O}_{\mathbf{4}}{ }^{3-}$ & $\mathbf{C F}$ & $\mathbf{S F}$ \\
\hline $\mathbf{T}$ & $\mathbf{1}$ & & & & & & & \\
$\mathbf{p H}$ & 0,971 & $\mathbf{1}$ & & & & & & \\
$\mathbf{C E}$ & 0,921 & 0,988 & $\mathbf{1}$ & & & & & \\
$\mathbf{C l}-$ & 0,988 & 0,996 & 0,970 & $\mathbf{1}$ & & & & \\
$\mathbf{N H}_{4}{ }^{+}$ & $-0,007$ & $-0,228$ & $-0,373$ & $-0,147$ & $\mathbf{1}$ & & & \\
$\mathbf{P O}_{\mathbf{4}}{ }^{-}$ & 0,046 & $-0,178$ & $-0,326$ & $-0,095$ & 0,998 & $\mathbf{1}$ & & \\
$\mathbf{C F}$ & 0,093 & $-0,036$ & $-0,138$ & 0,016 & 0,814 & 0,809 & $\mathbf{1}$ & \\
$\mathbf{S F}$ & 0,173 & 0,017 & $-0,101$ & 0,079 & 0,873 & 0,873 & 0,988 & $\mathbf{1}$ \\
\hline
\end{tabular}

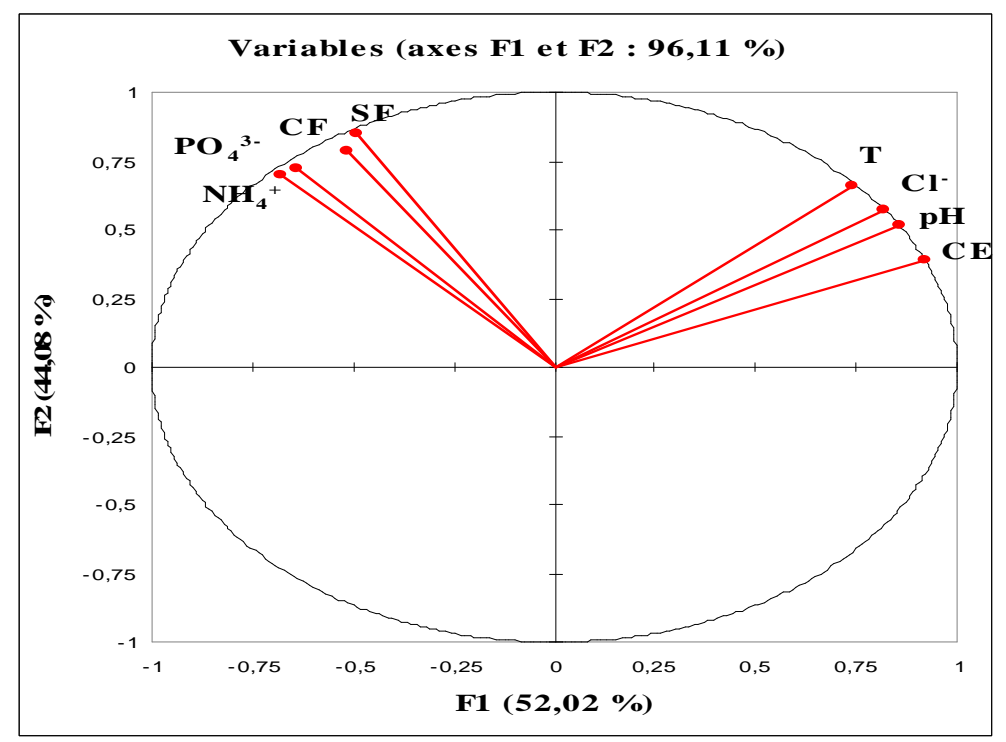

Figure 1: Projection des variables dans l'espace des axes F1et F2.

\section{DISCUSSION}

Les températures enregistrées au niveau des effluents de la ville de Nouakchott sont comprises entre $21,9{ }^{\circ} \mathrm{C}$ et $26,3{ }^{\circ} \mathrm{C}$ comme valeurs extrêmes minimales et maximales et $24,3{ }^{\circ} \mathrm{C}$ comme étant la température moyenne (Tableau 1). Les valeurs moyennes minimales et maximales de $\mathrm{pH}$ varient respectivement de 6,91 à 8,17 (Tableau 1). Cependant, l'effluent de la SOBOMA est caractérisé par une température oscillant entre $32,5{ }^{\circ} \mathrm{C}$ et $35,8^{\circ} \mathrm{C}$ et un $\mathrm{pH}$ variant entre 12,13 et 12,24 . La valeur maximale de la conductivité enregistrée au niveau des effluents de la ville de
Nouakchott est de $5,5 \mathrm{mS} / \mathrm{cm}$, et la valeur minimale est de $1,5 \mathrm{mS} / \mathrm{cm}$ (Tableau 1). Ces résultats pourraient être expliqués d'une part par le rejet des eaux usées résiduaires des unités industrielles connectées aussi à la STEP. Les effluents de la SOBOMA et de la Pro Pêche sont caractérisés par des valeurs de la conductivité oscillant respectivement de 4,4 $\mathrm{mS} / \mathrm{cm}$ à $5 \mathrm{mS} / \mathrm{cm}$ et de $29,7 \mathrm{mS} / \mathrm{cm}$ à 30,2 $\mathrm{mS} / \mathrm{cm}$. La valeur maximale en chlorures atteinte enregistrée au niveau des effluents de la ville de Nouakchott est de $1633 \mathrm{mg} /$ /litre et la valeur minimale est de $194 \mathrm{mg} / \mathrm{litre}$ (Tableau 1). L'eau potable de la ville de 
Nouakchott est caractérisée par des teneurs en chlorures oscillant entre $106,5 \mathrm{mg}$ /litre et $127,8 \mathrm{mg} /$ litre. On note une forte teneur en chlorures apportée par la Pro Pêche utilisant en grande partie de l'eau de mer pour le traitement des céphalopodes (poulpes et seiches). La présence des ions ammonium est à rapprocher à celle des autres éléments azotés identifiés dans l'eau (nitrate, nitrite) et des résultats de l'analyse bactériologique (Rodier, 1996). L'urine est la principale source d'ammonium dans les eaux usées domestiques (Udert et al., 2003 ; Bonté et al., 2008). La grande partie du phosphore organique provient des déchets du métabolisme des protéines et de son élimination sous forme de phosphates dans les urines par l'homme (Du Chaufour, 1997). La valeur moyenne maximale atteinte en ammonium au niveau des effluents de la ville de Nouakchott est de $292,05 \mathrm{mg} / \mathrm{litre}$ et la valeur moyenne minimale est de $119,14 \mathrm{mg} / \mathrm{litre}$ et les teneurs moyennes en orthophosphates oscillant entre 19,82 mg/litre et 39,15 mg/litre (Tableau 1).

Les teneurs moyennes en coliformes fécaux et en streptocoques fécaux dans les effluents de la ville de Nouakchott sont respectivement de l'ordre de $9,03.10^{4}$ UFC/100 ml et $9,24.10^{4} / 100 \mathrm{ml}$ (Tableau 2). Ces valeurs sont inférieures à celles rencontrées dans des effluents urbains et périurbains de Dakar par Niang (1996). Aussi, cette charge bactérienne dépasse celle préconisée par l'OMS pour les eaux d'irrigation et qui est de l'ordre de $10^{3}$ CFU/100 ml (OMS, 1986). La charge fécale est très faible au niveau de la Pro Pêche, cette faible charge est due probablement à la forte salinité de son effluent. La salinité est un facteur de stress très important que subissent les bactéries de pollution fécale dans le milieu salé, où la bactérie doit établir l'équilibre osmotique entre le milieu extérieur et son cytoplasme (Chedad et Assobhi, 2007). La charge fécale est très faible aussi au niveau de la SOBOMA, cette faible charge fécale est due probablement à la forte basicité de son effluent. L'augmentation du $\mathrm{pH}$ affecte l'abondance des coliformes fécaux c'est-à-dire que les $\mathrm{pH}$ basiques entraînent une nette diminution de la survie des coliformes fécaux (Mayo, 1995). L'origine de la pollution fécale est reliée au rapport quantitatif des coliformes fécaux sur les streptocoques fécaux (CF/SF). Lorsque ce rapport $\mathrm{CF} / \mathrm{SF}$ est inférieur à 0,7 la pollution a pour origine principalement ou entièrement humaine; si le rapport est compris entre 0,7 et 1 , dans ce cas, la pollution est mixte à prédominance animale. Le rapport $\mathrm{CF} / \mathrm{SF}$ compris entre 1 et 2 nous dit que l'origine est incertaine. Si le rapport $\mathrm{CF} / \mathrm{SF}$ est compris entre 2 et 4 , dans ce cas, la pollution est mixte avec prédominance humaine. Si le rapport $\mathrm{CF} / \mathrm{SF}$ est supérieur à 4, dans ce cas, la source est exclusivement humaine (Bricha et al., 2007). Le rapport $\mathrm{CF} / \mathrm{SF}$ est compris entre 0,7 et 1 (Tableau 2), ce qui laisse supposer que les effluents de la ville de Nouakchott sont contaminés par une pollution très probablement mixte (d'origine animale et humaine), mais à prédominance animale.

L'ACP est un outil d'analyse de données qui permet d'expliquer la structure des corrélations ou des covariances en utilisant des combinaisons linéaires des données originelles. Son utilisation permet de réduire et d'interpréter les données sur un espace réduit (Lagarde, 1995 ; Maliki, 2000). Une analyse en composante principale de l'ensemble des données obtenues sur les effluents de la ville de Nouakchott, prélevées lors des 13 campagnes d'échantillonnage, fait apparaître que le premier axe F1, qui explique $52,02 \%$ de la variance, est principalement dû à tous les paramètres bactériologiques mesurés, les ions ammonium, les ions orthophosphates (Tableau 3) et (Figure 1) alors que le deuxième axe F2, qui représente $44,08 \%$ de la variance, est marqué par la présence de la température, du $\mathrm{pH}$, de la conductivité et des ions chlorures (Tableau 3 et Figure 1). Des corrélations positives significatives ont été observées entre les coliformes fécaux et les ions ammonium, les coliformes fécaux et les ions orthophosphates. Des corrélations 
positives faibles ont été observées entre les coliformes fécaux et la température, les coliformes fécaux et les ions chlorures (Tableau 4). Des corrélations positives significatives ont été observées entre les streptocoques fécaux et les ions ammonium, les streptocoques fécaux et les ions orthophosphates. Des corrélations positives faibles ont été observées entre les streptocoques fécaux et la température, les streptocoques fécaux et le $\mathrm{pH}$, streptocoques fécaux et les ions chlorures (Tableau 4). On note aussi qu'une corrélation positive significative est observée entre les coliformes fécaux et les streptocoques fécaux (Tableau 4). La significative interconnexion des variables bactériologiques considérés dans cette étude montre que le suivi de l'un d'entre elle suffirait pour la caractérisation de la pollution bactériologique.

Les effluents de la Pro Pêche sont constitués par une forte salinité (conductivité et ions chlorures) due à l'utilisation de l'eau de mer pour le traitement des céphalopodes et l'effluent de la SOBOMA est caractérisé par des valeurs très élevées $\mathrm{du} \mathrm{pH}$ et de la température, c'est-à-dire une forte alcalinité due à l'utilisation de la soude caustique pour le rinçage des bouteilles et les températures des bains de rinçage des bouteilles se situant dans un intervalle de $20{ }^{\circ} \mathrm{C}$ à $80^{\circ} \mathrm{C}$ dans le cas de la SOBOMA. Ces effluents sont caractéristiques de l'axe F1. Les rejets domestiques proviennent des différents usages domestiques de l'eau et se répartissent en eaux ménagères, qui ont pour origine les salles de bains et les cuisines et sont généralement chargées de détergents, les rejets des toilettes, chargés de diverses matières organiques azotées et de germes fécaux correspondant au groupe des paramètres de l'axe F2.

\section{Conclusion}

Les niveaux de pollutions bactériologiques enregistrées au niveau des effluents de la ville de Nouakchott irrigués dans le périmètre maraîcher de Sebkha sont plus élevés que les seuils recommandés par l'O.M.S. Le rapport CF/SF est compris entre 0,7 et 1 , ce qui laisse supposer que les effluents de la ville de Nouakchott sont contaminés par une pollution très probablement mixte (d'origine animale et humaine) avec une prédominance animale. L'analyse en composante principale nous renseigne aussi que la pollution fécale est d'origine domestique. La présence très élevée des germes indicateurs de la contamination fécale, constitue sans doute une menace pour les exploitants maraîchers, les enfants des exploitants, les revendeurs et les consommateurs.

\section{REFERENCES}

Angulo FJ, Tippen S, Sharp DJ, Payne BJ, Collier C, Hill JE, Barret TJ, Clark RM, Geldreich EE, Donnel HD, Swerdlow DL. 1997. A community waterborne outbreak of salmonellosis and effectveness of a boil water order. American Journal of Public Health, 87(4): 580-584.

Azandosessi A, Ould Selmane ML, Ould Baba L, Benzeroug EH, Cissé G, Nner M. 1999. Projet de préservation de l'unique espace vert de Nouakchott : le site de Sebkha « Nouakchott El Khadra ». Document de projet. OMS, Nouakchott.

Balbus JM, Embrey MA. 2002. Risk Factors for Waterborne Enteric Infections. Current Opinion in Gastroenterology, 18(1): 46-50.

Bonté SL, Pons M, Potier O, Rocklin P. 2008. Relation between Conductivity and Ion Content in Urban Wastewater. Journal of Water Science, 21(4): 429- 438.

Bricha S, Ounine K, Oulkheir S, Attrassi B. 2007. Etude de la qualité physicochimique et bactériologique de la nappe phréatique M'nasra (Maroc). Afrique Science, 03(3) : 391 - 404.

Chedad K, Assobhi O. 2007. Etude de la survie des bactéries de contamination fécale (coliformes fécaux) dans les eaux de la zone ostréicole de la lagune 
d'Oualidia (Maroc). Bulletin de l'Institut Scientifique, Rabat, Section Sciences de la Vie, (29) : 79.

Du Chaufour P. 1997. Abrégé de Pédologie: Sol, Végétation et Environnement (5è édition). Masson.

George I, Servais P. 2002. Sources et dynamique des coliformes dans le basin de la Seine. Centre National de la Recherche Scientifique, programme PIREN-Seine1998-2001: Rapport de synthèse Paris, France, 46p.

Lagarde J. 1995. Initiation à l'Analyse des Données. Ed. Dunod: Paris; 157.

Maliki AM. 2000. Etude hydrologique hydro chimique et isotopique de la nappe profonde de Sfax (Tunisie). Thèse de Doctorat Fac. Sci. Sfax, 301.

Mayo AW. 1995. Modeling coliform mortality in waste stabilization ponds. $J$. Environ. Engineer, 121(2) : 140-152.

Mint El Bezeid F. 2007. Evaluation de risques Environnement qui menacent la zone côtière de Nouakchott et les solutions possibles (Mauritanie). Mémoire DESA, Faculté des Sciences, EL Jadida Université, Chouaib Doukkali, Maroc.
N'diaye AD, Kankou MOSAO, Sarr AD, Baidy L. 2009. Caractérisation physicochimique des eaux usées brutes de la ville de Nouakchott, Rev. Ivr. Sc. Technol., 14 : 97-109.

Niang S. 1996. Utilisation des eaux usées domestiques en maraîchères périurbaines à Dakar (Sénégal). Sécheresse, 7(3) : 212223.

OMS. 1986. Directives de la qualité de l'eau de boisson : critères d'hygiène et documentation à l'appui Genève. 2e Edition.

Ould Baba L. 1998. Projet de préservation de l'unique espace vert de Nouakchott : le site de Sebkha « Nouakchott El Khadra ». Rapport, Bureau OMS Nouakchott.

Rodier J. 1996. L'analyse de l'Eau Naturelle, Eaux Résiduaires, Eau de Mer ( $8^{\text {éme } e ́ d n) . ~}$ Denod : Paris ; 1383 p.

Udert KM, Larsen TA, Biébow M, Gujer W. 2003. Urea hydrolysis and precipitation dynamics in a urine-collecting system. Water Res., 37: 2571-2582. 